\title{
FACTIBILIDAD DE UN PROGRAMA DE MINDFULNESS EN PACIENTES ONCOLÓGICOS DEL PERÚ: UN ESTUDIO PILOTO ALEATORIZADO
}

\author{
MANOLETE S. Moscoso \\ https://orcid.org/0000-0001-8713-5749 \\ University of South Florida / Tampa, Estados Unidos \\ Universidad San Martín de Porres, Lima, Perú \\ María A. de la Puente \\ ttps://orcid.org/0000-0001-5365-8314 \\ ANA L. PÉREZ \\ https://orcid.org/0000-0002-0801-7128 \\ RAÚL GUZMÁN \\ https://orcid.org/0000-0002-9921-2024 \\ Universidad Católica de Santa María / Arequipa, Perú
}

Recibido: 20 de julio del 2019 / Aceptado: 11 de diciembre del 2019

doi: 10.26439/persona2020.n023(1).4832

\begin{abstract}
Resumen. El propósito de esta investigación fue evaluar la factibilidad de la intervención cognitiva basada en mindfulness ( $\mathrm{MBCl}$ ) para pacientes con cáncer de mama en la ciudad de Arequipa, Perú. Se seleccionaron 26 pacientes con esta enfermedad entre las edades de 37 a 73 años para el presente estudio piloto. Las participantes fueron asignadas aleatoriamente al programa de entrenamiento $\mathrm{MBCl}$ de seis semanas de duración o al grupo de control. Se utilizaron instrumentos psicométricos sobre estrés percibido y depresión para colectar los datos antes y después del entrenamiento de $\mathrm{MBCl}$. La adherencia y participación de las pacientes en el programa, así como sus reportes con base en el protocolo clínico después de la intervención, confirman la factibilidad de la utilización del programa $\mathrm{MBCl}$ en pacientes con cáncer de mama en el Perú. Los resultados indican que, en comparación con el grupo de control, las participantes en el programa $\mathrm{MBCl}$ reportaron reducciones significativas en el estrés percibido y la depresión.
\end{abstract}

Palabras clave: mindfulness / estrés percibido / depresión / cáncer de mama

Correo electrónico: mmoscoso@health.usf.edu 


\title{
FEASIBILITY OF A MINDFULNESS INTERVENTION PROGRAM IN CANCER PATIENTS IN PERU: A RANDOMIZED PILOT STUDY
}

\begin{abstract}
This research aimed to evaluate the feasibility of a mindfulness-based cognitive intervention $(\mathrm{MBCl})$ among breast cancer patients in the city of Arequipa, Peru. Twenty-six (26) female patients with breast cancer aged 37 to 73 years were screened for this pilot study. The participants were randomized to the six-week $\mathrm{MBCl}$ program or to the control group. Psychometric instruments to measure perceived stress and depression were used to collect the data before and after the $\mathrm{MBCl}$ program. Patients' adherence to and participation in the program, as well as the protocol-based medical reports issued after the interventions, demonstrate the feasibility of using the $\mathrm{MBCl}$ program in patients with breast cancer in Peru. The results show that, compared with the control group, the participants randomized to the $\mathrm{MBCl}$ program reported significant reductions in perceived stress and depression.
\end{abstract}

Keywords: mindfulness / perceived stress / depression / breast 


\section{INTRODUCCIÓN}

En el Perú, el cáncer de mama es la segunda neoplasia maligna más frecuente entre las mujeres, después del cáncer de cuello uterino. Este tipo de cáncer, de carácter impredecible, se manifiesta con mayor incidencia entre los 30 y 80 años, con un porcentaje más alto entre los 45 y 65 años, de acuerdo con el reporte del Ministerio de Salud del Perú (2013). En cuanto a su mortalidad, la incidencia bajó un $2 \%$ anual en mujeres mayores de 50 años entre 1999 y 2005. La adaptación psicológica y psicosocial al cáncer de mama está determinada por varios factores, tales como la percepción de la imagen corporal (Sánchez, 2015; Rivera y Vásquez, 2013) y el soporte familiar (Lazo, 2015).

El diagnóstico del cáncer de mama y la evolución de esta enfermedad generan con frecuencia un estado de distrés emocional, porque afectan al paciente física, psíquica y socialmente (Hernández, Bernardello, Aristodemo y Barros, 2007; Tateishi, 2011). Por este motivo, la adaptación del paciente a la experiencia del cáncer es difícil, y provoca reacciones ansiosas y depresivas (Lengacher et al., 2009). Muchos de estos síntomas psicológicos no reciben tratamiento apropiado, al no ser evaluados, y suelen considerarse reacciones normales a la enfermedad y sus tratamientos (Moscoso, Lengacher y Reheiser, 2012).

\section{Afrontamiento del paciente oncológico ante el estrés crónico}

Una de las formas más comunes de afrontar el estrés en los pacientes con diagnóstico de cáncer es la internalización, es decir, una reacción inhibitoria ante el estrés (Chinchilla, 2008). Ello genera que la persona mantenga un nivel de excitación fisiológica y que las hormonas del estrés, como el cortisol, continúen haciendo estragos en el organismo. En este sentido, es importante destacar que cada vez hay más evidencias de que la estimulación crónica del sistema nervioso simpático puede llevar al individuo a un estado de desregulación neurofisiológica que cause aún mayores problemas en la salud de dichos pacientes (Moscoso, 2014). A la fecha, existen reportes científicos que indican que una situación estresante altera el sistema nervioso central, el cual a su vez afecta a los sistemas inmunológico y endocrino, debido a las múltiples conexiones mente-cuerpo (Molina, 2009; Moscoso, 2009; Moscoso, 2010).

Lazarus y Folkman (1986) indican que "el estrés psicológico es una relación particular entre el individuo y su entorno, el cual es evaluado como una amenaza a sus recursos personales poniendo en peligro su bienestar" (p. 43); aún más, el afrontamiento es "el proceso a través del cual el individuo maneja las demandas de la relación individuo-ambiente evaluando al estresor y las emociones que este genera" (Lazarus y Folkman, 1986, p. 44). La respuesta emocional al estrés está condicionada por la percepción que tenga la persona de tal situación, así como por sus características personales. 
En este sentido, la práctica del mindfulness permite que el paciente sea capaz de manejar el estrés crónico (Tacón, Caldera y Ronaghan, 2004) a través de enfocar la atención en la respiración y tomar conciencia de los pensamientos y emociones, al igual que mediante los sentidos (Stahl y Goldstein, 2010).

\section{Reducción de estrés basada en mindfulness}

En los últimos años, el interés en la reducción del estrés basada en mindfulness ha crecido exponencialmente, y se utiliza también en el manejo del distrés emocional asociado a enfermedades crónicas (Bishop, 2002; Kabat-Zinn, 2003). La práctica de mindfulness fue introducida por Jon Kabat-Zinn en 1979 en los Estados Unidos de América, con un programa formal de reducción de estrés que se llevó a cabo en el Centro Médico de la Universidad de Massachusetts, llamado Mindfulness Based Stress Reduction (MBSR).

Kabat-Zinn (2007) define mindfulness como "la toma de conciencia sin elaborar juicios de valor, el cual se cultiva enfocando la atención en el momento presente" (p. 115). Para Moscoso y Lengacher (2015), "es la energía consciente de prestar atención deliberadamente a la experiencia del momento presente, sin elaborar juicios de valor". Como podemos observar, existen diversas definiciones del término; sin embargo, se puede indicar que todas coinciden con el enfoque de la atención en el momento presente, es decir, en el aquí y el ahora (Mañas, 2009).

Speca, Carlson, Goodey y Angen (2000) afirman que la práctica de mindfulness es eficaz en el manejo de enfermedades físicas y psicológicas. Por ello la psicología contemporánea ha acogido el entrenamiento de mindfulness como una intervención psicológica válida y efectiva para afrontar de manera más adecuada los procesos de estrés. Igualmente, Lengacher et al. (2014) reportan evidencia empírica de que las intervenciones de mindfulness contribuyen a mejorar la capacidad de regular las emociones y disminuir los pensamientos negativos, además de ayudar a que la respuesta inmunitaria, la reactividad al estrés y la percepción de bienestar físico funcionen mejor. La eficacia de estas intervenciones ha sido también demostrada en el tratamiento de la depresión (Teasdale et al., 2000), la reducción del estrés y la ansiedad (Kabat-Zinn, 2003), así como del distrés psicológico en pacientes con cáncer (Carlson, Speca, Patel y Faris, 2007; Lengacher et al., 2009; Moscoso, Reheiser y Hann, 2004) y los temores de recurrencia del tumor maligno (Lengacher et al., 2014).

En términos generales, los efectos positivos de las intervenciones basadas en mindfulness también han sido demostrados en muestras no clínicas, específicamente en grupos de personas interesadas en la reducción del estrés y del dolor crónico (KabatZinn, 2007), la mejora de las funciones inmunológicas (Davidson et al., 2003; Lengacher et al., 2012) y disminución en los niveles de cortisol en pacientes con cáncer de mama 
(Lengacher et al., 2012). Existe también evidencia empírica de que este tipo de intervención facilita el desarrollo de actitudes y emociones positivas en estudiantes universitarios (Garland, Gaylord y Fredrickson, 2011).

\section{Intervención cognitiva basada en mindfulness}

El programa de intervención cognitiva basada en mindfulness ( $\mathrm{MBCl}$ ) fue desarrollado específicamente para pacientes oncológicos en 1998, a partir del programa MBSR de Jon Kabat-Zinn. La adaptación se llevó a cabo en el Morton Plant Hospital de Clearwater, Florida, por Manolete S. Moscoso (Moscoso et al., 2004). El MBCl tiene una duración de seis semanas y es relativamente comparable al programa MBSR de ocho semanas de Jon Kabat-Zinn. Ofrece entrenamiento en la reducción del estrés crónico, la respuesta de relajación y la modificación de patrones afectivos negativos. Asimismo, busca promover la calidad de vida del paciente con cáncer a través del desarrollo de un estilo de vida saludable (Moscoso, 2010). El MBCI orientado a la investigación científica se inició en el 2003 con apoyo económico de la Fundación Susan G. Komen, teniendo como propósito ayudar a los pacientes con cáncer en la reducción del estrés asociado al diagnóstico de la enfermedad y sus tratamientos respectivos.

A pesar de la evidencia científica acerca de la efectividad de mindfulness en la reducción del estrés, ansiedad y depresión de pacientes con cáncer, en el Perú no existen estudios empíricos que lo corroboren. Por esta razón, nuestro interés en llevar a cabo un estudio empírico piloto en esta línea de investigación ha sido prioritario, con el propósito de contribuir a mejorar la calidad de vida de los pacientes que padecen esta enfermedad en la ciudad de Arequipa. Nuestra hipótesis de trabajo plantea que el programa $\mathrm{MBCl}$ reduce los niveles de estrés percibido y depresión en los pacientes con cáncer de mama del Hospital Nacional Carlos Alberto Seguín Escobedo y del Instituto Regional de Enfermedades Neoplásicas del Sur de la ciudad de Arequipa. En este sentido, el objetivo principal de este trabajo es evaluar la factibilidad de este tipo de estudios en el Perú y a la vez examinar el nivel de efectividad del programa $\mathrm{MBCl}$, considerando los niveles de estrés percibido y depresión en pacientes con cáncer de mama de los nosocomios antes mencionados en la ciudad de Arequipa, Perú.

\section{MÉTODO}

\section{Participantes}

La muestra de la presente investigación estuvo conformada por un total de 26 mujeres que recibían tratamiento para el cáncer de mama en el Hospital Nacional Carlos Alberto Seguín Escobedo (HNCASE) y en el Instituto Regional de Enfermedades Neoplásicas (IREN) de Arequipa. Los criterios de inclusión fueron los siguientes: sexo femenino; 
diagnóstico de cáncer de mama en estadios I, II, y III; rango de edades entre 30 y 80 años, y estar recibiendo tratamiento médico durante el período de estudio. Todas las participantes fueron admitidas en la investigación de manera voluntaria. Las características demográficas de la muestra indican que las edades de las participantes oscilaban entre los 37 a 73 años, con un promedio de edad de 56 años. El $59 \%$ de las pacientes eran casadas, $29 \%$ solteras y $12 \%$ convivientes. Respecto al grado de instrucción, el $41 \%$ tenía educación superior, el 35 \% educación secundaria, el 18 \% educación primaria y el $6 \%$ reportó educación técnica. Con relación a la ocupación actual de las pacientes, se observó que el $70 \%$ de ellas eran amas de casa, el $6 \%$ artesanas, el $6 \%$ profesoras, el $6 \%$ costureras, el $6 \%$ contadoras y las demás señalaron ser trabajadoras independientes. En referencia al estadio de la enfermedad, el $24 \%$ de las participantes estaban en el estadio I de cáncer de mama, el $64 \%$ presentó un estadio II y el $12 \%$ se encontraba en un estadio III. En cuanto al tipo de tratamiento, las participantes recibían tratamiento médico en la forma de quimioterapia, radioterapia, hormonoterapia y/o mastectomía.

\section{Diseño y procedimiento}

Con el propósito de iniciar la recolección de datos, se solicitó autorización escrita al Hospital Nacional Carlos Alberto Seguín Escobedo (HNCASE) y al Instituto Regional de Enfermedades Neoplásicas del Sur (IREN) para acceder a la muestra respectiva; asimismo, se pidió hacer uso de los ambientes de quimioterapia ambulatoria y radioterapia de ambos hospitales. En el marco de las reuniones de orientación, se invitó a las pacientes a participar en el estudio. Las interesadas en nuestro programa recibieron el consentimiento informado, indicándoles que su participación era de carácter voluntario.

Siguiendo las pautas metodológicas de Hernández, Fernández y Baptista (2010), la presente investigación es de tipo experimental con el modelo de medidas repetidas pretest y postest. Se utilizó el diseño aleatorizado con un grupo experimental y un grupo de control, aplicando el método de asignación aleatoria simple para distribuir a las pacientes entre ambos grupos. Este procedimiento se realizó por medio de un sorteo, en el cual las participantes tenían la opción de obtener un número par o impar. Las que obtuvieron un número par fueron asignadas al grupo experimental, y quienes sacaron un número impar fueron colocadas en el grupo de control. La distribución de grupos aleatorizados, reconocida como el método gold standard de muestreo estadístico bajo condiciones razonables, asegura estadísticamente que dos o más grupos de intervención son equivalentes entre sí, con la intención de controlar variables que confabulen en los resultados de la investigación. El muestreo aleatorio para la conformación de los grupos se realizó en cada hospital por separado. Así, 14 participantes formaron el grupo experimental y 12 , el grupo de control. 
Una vez distribuidos los grupos, se procedió a realizar la evaluación del pretest, que incluye la ficha de datos personales y las pruebas psicométricas. Durante el desarrollo del programa se llevó un registro de asistencia de las pacientes y, una vez culminadas las seis semanas del programa $\mathrm{MBCl}$, se procedió a efectuar la evaluación postest a todas las participantes del grupo experimental y de control. Finalmente, se ofreció el programa $\mathrm{MBCl}$ gratuitamente al grupo de control por razones éticas y de cortesía a su participación voluntaria. Las sesiones formales del estudio se llevaron a cabo durante el período comprendido entre los meses de octubre, noviembre y diciembre del año 2016. Cabe indicar que durante el desarrollo del programa se retiraron cuatro pacientes del grupo experimental y dos pacientes del grupo de control, debido a complicaciones de la enfermedad y los efectos secundarios de los tratamientos. Asimismo, se perdió contacto con tres pacientes del grupo de control, de manera que la muestra quedó finalmente reducida a 17 participantes (65,38\%), quienes culminaron el programa $\mathrm{MBCl}$ de seis semanas. La población de pacientes oncológicos es muy frágil; por lo tanto, es común que ocurra este tipo de deserción.

La implementación del programa $\mathrm{MBCl}$ de seis semanas tuvo como objetivo fomentar en las pacientes el desarrollo de sus habilidades para afrontar el estrés relacionado con el dolor y el diagnóstico de cáncer, pero también se consideró el aprendizaje de habilidades que les ayudaran a mejorar su salud, bienestar personal y calidad de vida. La intervención se llevó a cabo de acuerdo con el manual clínico y sílabo establecido por Manolete Moscoso (Moscoso et al., 2004). Se realizó una sesión por semana con una duración de 2 horas. Asimismo, cada paciente hizo tareas en casa que incluían un mínimo de 30 minutos por día de práctica de mindfulness formal y 30 minutos de práctica informal, por 5 días a la semana en toda la duración del curso. En la primera semana, se entregó un CD a las pacientes, el cual contenía la práctica de la meditación sentada, y un cuadernillo con fichas para completar día a día su práctica formal e informal de mindfulness. En la segunda semana, se les dio un CD que incluía la práctica del escaneo corporal. En la tercera semana, se les repartió un tríptico de práctica de yoga para pacientes con diagnóstico de cáncer. En la quinta semana, se les proporcionó un CD con la práctica de la meditación de la montaña.

\section{Instrumentos de evaluación}

La ficha demográfica. Contiene información básica sobre cada una de las participantes: nombre, fecha de nacimiento, grado de instrucción, estado civil y situación laboral actual. La segunda parte incluye datos médicos, como el diagnóstico, tiempo e inicio de la enfermedad, estadio actual del tumor, pronóstico y complicaciones relacionadas con los tratamientos recibidos. 
El Inventario de Depresión de Beck II (BDI-II, Beck, Steer y Brown, 1996). Es una prueba que consta de 21 reactivos, cuyo propósito es medir la presencia y severidad de la depresión. Está dirigida a adultos y adolescentes, a partir de 13 años de edad en adelante. Presenta cuatro alternativas de respuesta para cada ítem, los cuales evalúan la intensidad de los síntomas. La puntuación total fluctúa entre 0 y 3; a mayor puntuación en el inventario, mayor es la sintomatología depresiva. El contenido de la prueba enfatiza en los componentes cognitivo-afectivos y somáticos de la depresión. El rango de puntuación varía de 0 a 63 puntos. Los puntos de corte usualmente aceptados para graduar la intensidad de la depresión son los siguientes: de 0 a 13 se encuentra en el rango de depresión mínima, de 14 a 19 está en el rango de depresión leve, de 20 a 28 pertenece a la categoría de depresión moderada y de 29 a 63 corresponde a una depresión severa. En la población peruana, el BDI-Il se ha utilizado en las investigaciones epidemiológicas del Instituto de Salud Mental Honorio Delgado - Hideyo Noguchi. Tateishi (2011) analizó la confiabilidad por consistencia interna de este instrumento y obtuvo un alfa de Cronbach de 0,89. En el análisis de la capacidad discriminativa de los ítems a partir de las correlaciones ítem-test, los coeficientes fluctuaron entre 0,26 y 0,75. La confiabilidad por consistencia interna del factor cognitivo-afectivo reporta un alfa de Cronbach de 0,87 con correlaciones ítem-test mayores a 0,29. Para el factor somático, el alfa de Cronbach fue de 0,63 y las correlaciones ítem-test mayores a 0,35.

La Escala de Estrés Percibido. Es un instrumento psicométrico que evalúa el nivel de estrés percibido durante el último mes. En su versión 2.0 de 14 ítems fue adaptado al idioma español y tiene una confiabilidad de 0,81 (Roldán y Torres, 2009). La escala obtiene información sobre la percepción de la persona en cuanto a las dificultades de la vida diaria, situaciones de conflicto o fuera de control. Fue elaborada sobre la base de síntomas de estrés de tipo fisiológico, emotivo y cognitivo. La calificación de la escala se realiza a través de una escala Likert de cinco puntos: donde 1 es "nunca", 2 "casi nunca", 3 "de vez en cuando", 4 "frecuentemente", 5 "muy frecuente". Los ítems 4, 5, 6, 7, 9, 10 y 13 son inversos, por lo que se necesita invertir las puntuaciones en el momento de la calificación. La puntuación máxima que se puede alcanzar en la escala es de 70 puntos y la mínima es de 14 puntos (García, 2012, pp. 41-42). Los rangos de clasificación indican que un puntaje de 14-31 representa un nivel bajo de estrés percibido, de 32-52 equivale a un nivel medio de estrés percibido y, finalmente, de 53-70 significa un nivel elevado de estrés percibido, donde la percepción de aceleraciones fisiológicas, tensión y rigidez muscular en cuello y espalda son muy frecuentes, y se presentan en la mayoría de situaciones adversas. Las personas en este rango registran pensamientos negativos y pesimistas de sí mismos, y de la vida. Sus sensaciones de inseguridad y desconfianza son frecuentes, así como el temor ante las dificultades de la vida diaria, situaciones conflictivas o fuera de control (Roldán y Torres, 2009, p. 33). 


\section{El programa de intervención}

El programa $\mathrm{MBCl}$ se puede describir brevemente de la siguiente manera. La primera semana se dedica a la práctica de enfocar la atención en la respiración, así como al aprendizaje del control y sostenimiento de la atención en la respiración (mindful breathing). Durante la segunda sesión, se entrena a los pacientes en el manejo de la exploración corporal guiada y visualización (body scan). En la tercera semana, se practica y enfoca la atención en la respuesta de relajación (relaxation response), la observación y percepción individual de estresores cotidianos (appraisal), así como el aprendizaje de la técnica de decentering. La cuarta semana tiene como meta el aprendizaje y toma de conciencia de la activación del nervio vago (vagus nerve awareness) a través de la respuesta de relajación. Asimismo, se continúa con la práctica de exploración corporal guiada y las diferentes posturas de yoga (gentle yoga) para pacientes con diagnóstico de cáncer. En la quinta sesión, se realiza el aprendizaje sobre los procesos de reevaluación cognitiva (positive reappraisal), relacionados con la toma de conciencia de los patrones habituales de pensamientos y emociones negativas experimentados por el paciente. Finalmente, la sexta semana está dirigida a la integración de la práctica de mindfulness en el contexto del estrés crónico, incluyendo los principios de aceptación y compasión (Moscoso et al., 2004; Moscoso, 2010).

\section{Feedback positivo}

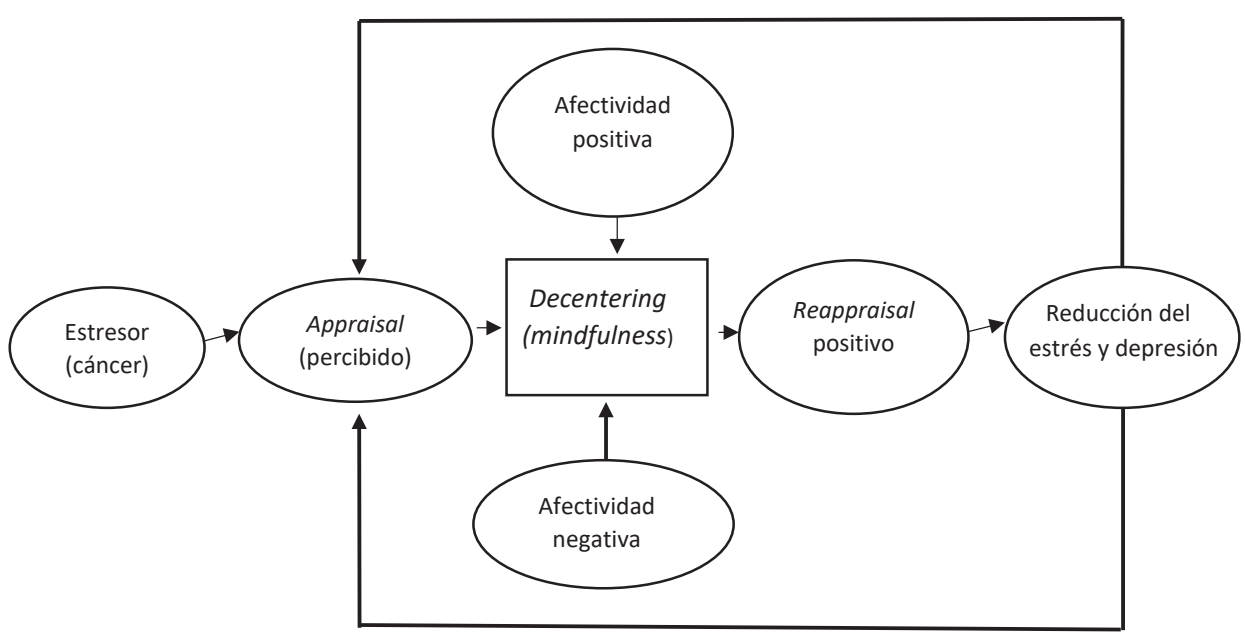

Feedback positivo

Figura 1. Marco conceptual de la intervención cognitiva basada en el mindfulness 


\section{Análisis de datos}

Una vez culminadas las evaluaciones de las participantes, se procedió a vaciar los datos en el programa estadístico SPSS versión 21. Con el propósito de examinar el efecto del programa de mindfulness en el grupo experimental y el grupo de control, se llevaron a cabo los análisis descriptivos para observar las medias, las desviaciones estándar y los porcentajes de las variables de estrés percibido y depresión del pretest y postest. Se utilizó el estadístico t para muestras relacionadas con la intención de observar las diferencias de las medias del estrés percibido y depresión en el pretest y postest de las participantes. En el análisis de los datos se utilizó un p-value convencional de .05 para examinar la significancia estadística de la intervención de mindfulness. Igualmente, se empleó el método d de Cohen para examinar la magnitud del efecto en los grupos experimental y de control, considerando las variables de estrés percibido y depresión.

\section{RESULTADOS}

Los análisis descriptivos de las medias y porcentajes del estrés percibido y depresión obtenidos en el pretest se presentan en la tabla 1. Igualmente, las medias y porcentajes del estrés percibido y depresión observados en el postest se describen en la tabla 2. En la tabla 1, se puede apreciar que el $100 \%$ de las pacientes del grupo experimental evaluadas reportaron un nivel moderado de estrés percibido, mientras que los niveles de depresión fluctuaron entre leve (20\%), moderado (50 \%) y severo (30\%) en las evaluaciones de pretest. Asimismo, en el pretest del grupo de control, el $100 \%$ de las pacientes tenían un nivel moderado de estrés percibido. En cuanto a la depresión en este grupo, el $42,9 \%$ de las pacientes manifestaron un nivel de depresión mínimo; el 14,3\%, depresión leve; y el $14,3 \%$, una depresión moderada; mientras que el $28,6 \%$ de las participantes experimentaron depresión severa. Las medias de estrés percibido observadas en las evaluaciones de pretest son relativamente similares: 41,3 para el grupo experimental y 39,0 para el grupo de control. Las medias de depresión fueron de 27,3 para el grupo experimental y de 18,2 para el grupo de control en estas evaluaciones de pretest.

Tabla 1

Pretest: niveles de estrés percibido y depresión en los grupos de control y experimental

\begin{tabular}{lcccc}
\hline $\begin{array}{l}\text { Niveles de estrés percibido } \\
\text { (SSP) }\end{array}$ & \multicolumn{2}{c}{$\begin{array}{c}\text { Grupo de control } \\
\mathrm{M}=39,0\end{array}$} & \multicolumn{2}{c}{$\begin{array}{c}\text { Grupo experimental } \\
\end{array}$} \\
\cline { 2 - 5 } & $(\mathrm{N}=7)$ & Porcentaje & $(\mathrm{N}=10)$ & Porcentaje \\
\hline Estrés percibido bajo & 0 & 0 & 0 & 0 \\
Estrés percibido moderado & 7 & $100 \%$ & 10 & $100 \%$ \\
Estrés percibido alto & 0 & 0 & 0 & 0 \\
& & & & (continúa)
\end{tabular}


(continuación)

\begin{tabular}{lcccc}
\hline $\begin{array}{l}\text { Niveles de depresión } \\
\text { (BDI-II) }\end{array}$ & \multicolumn{2}{c}{$\begin{array}{c}\text { Grupo de control } \\
M=18,2\end{array}$} & \multicolumn{2}{c}{$\begin{array}{c}\text { Grupo experimental } \\
\end{array}$} \\
\cline { 2 - 4 } & $(\mathrm{N}=7)$ & Porcentaje & $(\mathrm{N}=10)$ & Porcentaje \\
\hline Depresión mínima & 3 & $42,9 \%$ & 0 & 0 \\
Depresión leve & 1 & $14,3 \%$ & 2 & $20,0 \%$ \\
Depresión moderada & 1 & $14,3 \%$ & 5 & $50,0 \%$ \\
Depresión severa & 2 & $28,6 \%$ & 3 & $30,0 \%$ \\
\end{tabular}

BDI-II = Beck Depression Inventory II

La tabla 2 muestra las evaluaciones de postest, donde se observa que el $20 \%$ de las pacientes del grupo experimental redujeron sus niveles de estrés percibido, mientras que solo el $14,3 \%$ del grupo de control tuvo una reducción de esta variable. Los resultados de postest relacionados con la depresión en el grupo experimental indican que el $50 \%$ de las pacientes redujeron sus estados depresivos a niveles mínimos y el 30 \% llegó a un nivel leve en la prueba de Beck, pero el 20 \% continuó experimentando una depresión severa. En el postest del grupo de control, se aprecia que el 42,9\% de la muestra reportó una depresión moderada y el 57,1 \% indicó la experiencia de una depresión mínima. Los puntajes medios de estrés percibido hallados en el postest presentan ciertas diferencias: 35,7 en el grupo experimental y 39,0 en el grupo de control. En cuanto a los niveles de depresión, las medias son similares para ambos grupos: 16,3 para el grupo experimental y 16,0 para el grupo de control. Cabe señalar que el grupo experimental tuvo una reducción de 11 puntos en la prueba de Beck, en comparación con las evaluaciones de pretest; en cambio, el grupo de control logró disminuir únicamente 2,2 puntos (véase la tabla 1).

Tabla 2

Postest: niveles de estrés percibido y depresión de los grupos de control y experimental

\begin{tabular}{|c|c|c|c|c|}
\hline \multirow{2}{*}{$\begin{array}{l}\text { Niveles de estrés percibido } \\
\qquad \text { (SSP) }\end{array}$} & \multicolumn{2}{|c|}{$\begin{array}{l}\text { Grupo de control } \\
\qquad M=39,0\end{array}$} & \multicolumn{2}{|c|}{$\begin{array}{l}\text { Grupo experimental } \\
\qquad M=35,7\end{array}$} \\
\hline & $(N=7)$ & Porcentaje & $(N=10)$ & Porcentaje \\
\hline Estrés percibido bajo & 1 & $14,3 \%$ & 2 & $20 \%$ \\
\hline Estrés percibido moderado & 6 & $85,7 \%$ & 8 & $80 \%$ \\
\hline Estrés percibido alto & 0 & 0 & 0 & 0 \\
\hline
\end{tabular}


(continuación)

\begin{tabular}{lcccc}
\hline Niveles de depresión & \multicolumn{2}{c}{ Grupo de control } & \multicolumn{2}{c}{ Grupo experimental } \\
(BDI-II) & \multicolumn{2}{c}{$\mathrm{M}=16,0$} & \multicolumn{2}{c}{$\mathrm{M}=16,3$} \\
\cline { 2 - 5 } & $(\mathrm{N}=7)$ & Porcentaje & $(\mathrm{N}=10)$ & Porcentaje \\
\hline Depresión mínima & 4 & $57,1 \%$ & 5 & $50 \%$ \\
Depresión leve & 0 & 0 & 3 & $30 \%$ \\
Depresión moderada & 3 & $42,9 \%$ & 0 & 0 \\
Depresión severa & 0 & 0 & 2 & $20 \%$ \\
& & & &
\end{tabular}

BDI-II = Beck Depression Inventory II

Los resultados acerca del nivel de significancia estadística relacionados con las dos variables psicológicas (estrés percibido y depresión) se presentan en la tabla 3. Como se puede apreciar, en primer lugar, el grupo experimental tuvo una reducción significativa del estrés percibido $(p=.01)$, con base en el valor estadístico $t=2,87$ y el intervalo de confianza de $95 \%$ (IC), 1,18-10,01. De igual manera, se observa una reducción significativa de la depresión $(p=.01)$, en función del valor estadístico $t=3,01$ y del intervalo de confianza de $95 \%$ (IC), 2.71-19.08 en el grupo experimental. El programa MBCI muestra una magnitud del efecto que es superior al del grupo de control, tanto para el estrés percibido como para la depresión. De acuerdo con el análisis del método d de Cohen, se observa un efecto relativamente grande en el caso del estrés percibido $(d=1,1)$, al igual que en los niveles de depresión $(\mathrm{d}=0,90)$.

Tabla 3

Efectos del programa de intervención cognitiva basada en mindfulness: grupo experimental

\begin{tabular}{lcccccc}
\hline Media & \multicolumn{3}{c}{ Diferencias relacionadas de pretest y postest } & & p-value \\
\cline { 2 - 5 } & Media & $\begin{array}{c}\text { Desviación } \\
\text { estándar }\end{array}$ & $\begin{array}{c}95 \% \text { de intervalo de confianza } \\
\text { para la diferencia }\end{array}$ \\
\hline $\begin{array}{l}\text { Estrés } \\
\text { percibido }\end{array}$ & 5,60 & 6,16 & 1,18 & 10,01 & 2,87 & 0,018 \\
Depresión & 11,00 & 11,44 & 2,71 & 19,08 & 3,01 & 0,015 \\
\hline
\end{tabular}

$p<.05$

La tabla 4 muestra los resultados obtenidos en función de las diferencias de las medias, desviaciones estándar e intervalos de confianza al $95 \%$ del pretest y postest del grupo de control, así como también los valores t y nivel de significancia (p) tanto para el constructo de estrés percibido como para la depresión. 
Tabla 4

Efectos del programa de intervención cognitiva basada en mindfulness: grupo de control

\begin{tabular}{|c|c|c|c|c|c|c|}
\hline \multirow[t]{3}{*}{ Media } & \multicolumn{4}{|c|}{ Diferencias relacionadas de pretest y postest } & \multirow[t]{3}{*}{ t } & \multirow[t]{3}{*}{ p-value } \\
\hline & \multirow[t]{2}{*}{ Media } & \multirow[t]{2}{*}{$\begin{array}{l}\text { Desviación } \\
\text { estándar }\end{array}$} & \multicolumn{2}{|c|}{$\begin{array}{c}95 \% \text { de intervalo de confianza } \\
\text { para la diferencia }\end{array}$} & & \\
\hline & & & Inferior & Superior & & \\
\hline $\begin{array}{l}\text { Estrés } \\
\text { percibido }\end{array}$ & 0,00 & 3,78 & $-3,50$ & $-0,50$ & 0,00 & 1,00 \\
\hline Depresión & 2,21 & 8,90 & 5,94 & 1,51 & 0,67 & 0,52 \\
\hline
\end{tabular}

$p<.05$

De acuerdo a la tabla anterior para la pre y posprueba del grupo experimental, en la variable estrés percibido el valor de $t=2,871$ y su significancia es de .018 , además nos da el intervalo de confianza.

\section{DISCUSIÓN Y CONCLUSIONES}

Por tratarse de la primera vez que se lleva a cabo un programa de intervención de esta naturaleza en el Perú, el propósito principal del presente estudio fue observar el nivel de factibilidad de su ejecución en pacientes oncológicos. Es de conocimiento general que las restricciones administrativas, recolección de datos, financiamiento, disponibilidad y aceptación de las pacientes con diagnóstico de cáncer son barreras que limitan el avance de la investigación científica intervencional en nuestro país. En este sentido, el objetivo central se logró al llevar a cabo las evaluaciones y análisis estadísticos sobre la base de un $65,38 \%$ de adherencia de las participantes al protocolo clínico del programa. A pesar del éxito inicial observado, el estudio debe ser considerado esencialmente de carácter exploratorio, en la forma de un plan piloto, con la noción de evaluar las posibilidades de continuar esta línea de investigación psicológica en el futuro.

De igual manera, se tuvo como interés examinar el nivel de significancia estadística y la magnitud del efecto del programa de mindfulness (MBCl) en pacientes con cáncer de mama en la región de Arequipa. Específicamente, este propósito estuvo centrado en observar el posible impacto de este modelo particular de intervención cognitiva en la reducción del estrés percibido y de la depresión. Podemos concluir que los resultados de la presente investigación, según el nivel de significancia estadística ( $p$-value) y la magnitud del efecto (d de Cohen), confirman la hipótesis inicialmente planteada: el programa de mindfulness $(\mathrm{MBCl})$ reduce los niveles de estrés percibido y de depresión 
en pacientes con cáncer de mama. Estos resultados preliminares observados en la presente muestra peruana coinciden con los hallazgos de Speca et al. (2000) en Estados Unidos de América, quienes reportaron una reducción del estrés del 31 \%. De la misma manera, comprueban que la utilización de este tipo de programas de intervención basados en mindfulness son favorables para disminuir la depresión en pacientes con cáncer. Esto corrobora los resultados de otros estudios llevados a cabo con este mismo tipo de pacientes (Lengacher et al., 2009). Es decir, con respecto al grupo de control, las pacientes asignadas al programa de mindfulness $(\mathrm{MBCl})$ de nuestro estudio alcanzaron niveles significativamente más bajos de depresión después de su participación en el programa de seis semanas de duración.

La presente investigación fue llevada a cabo con base en un riguroso diseño metodológico, con un muestreo aleatorizado que es considerado el modelo gold standard para estudios de intervención psicológica en investigación científica. Con ella se demuestra que este tipo de investigaciones son factibles en nuestro país, a pesar de las barreras logísticas y de apoyo financiero. Sin embargo, cabe indicar que los resultados reportados en esta investigación presentan limitaciones en términos del tamaño de la muestra ( $\mathrm{n}=$ 26). En todo caso, esta limitación es razonable y está justificada por el hecho de ser un estudio piloto y de naturaleza exploratoria. Sugerimos que la planificación de futuros estudios de intervención basada en mindfulness tome en cuenta una muestra más amplia con la intención de controlar la posibilidad de errores de tipo I. De la misma manera, sería recomendable aplicar este tipo de investigaciones de intervención basada en la práctica de mindfulness a otras formas de cáncer, así como también a otro tipo de poblaciones con diferentes trastornos psicológicos.

\section{REFERENCIAS}

Beck, A., Steer, R., y Brown, G. (1996) Inventario de Depresión de Beck II, segunda edición. Buenos Aires: Paidós.

Bishop, S. R. (2002). What do we really know about mindfulness-based stress reduction? Psychosomatic Medicine, 64(1), 71-83.

Carlson, L. E., Speca, M., Patel, K. D., y Faris, P. (2007). One year pre-post intervention follow-up of psychological, immune, endocrine and blood pressure outcomes of mindfulness-based stress reduction (MBSR) in breast and prostate cancer outpatients. Brain, Behavior and Immunology, 21, 1038-1049.

Chinchilla, A. (2008). La depresión y sus máscaras. Aspectos terapéuticos. Madrid: Panamericana.

Davidson, R. J., Kabat-Zinn, J., Schumacher, J., Rosenkranz, M., Muller, D., Santorelli, S. F., y Sheridan, J. F. (2003). Alterations in brain and immune function produced by mindfulness meditation. Psychosomatic Medicine, 65, 564-570. 
García, P. (2012). Técnica de liberación emocional sobre el estrés percibido en pacientes con diabetes mellitus tipo II (tesis de pregrado). Universidad Católica de Santa María.

Garland, E. L., Gaylord, S. A., y Fredrickson, B. L. (2011). Positive appraisal mediates the stress-reductive effects of mindfulness: an upward spiral process. Mindfulness, 2, 59-67.

Hernández, G. A., Bernardello, E. T., Aristodemo, J., y Barros, A. C. (2007). Cáncer de mama. Caracas: McGraw-Hill.

Hernández, R., Fernández, C., y Baptista, P. (2010). Metodología de la investigación. México D. F.: McGraw-Hill.

Kabat-Zinn, J. (2003). Vivir con plenitud las crisis. Cómo utilizar la sabiduría del cuerpo y de la mente para afrontar el estrés, el dolor y la enfermedad. Barcelona: Kairós.

Kabat-Zinn, J. (2007). La práctica de la atención plena. Barcelona: Kairós.

Lazarus, R. S., y Folkman, S. (1986). Estrés y procesos cognitivos. Barcelona: Martínez Roca.

Lazo, A. (2015). Dinámica familiar y depresión en pacientes oncológicos con quimioterapia del IREN (tesis de pregrado). Universidad Católica de Santa María.

Lengacher, C. A., Johnson-Mallard, V., Post-White, J., Moscoso, M. S., Jacobsen, P. B., Klein, T., et al. (2009). Randomized controlled trial of mindfulness-based stress reduction (MBSR) for survivors of breast cancer. Psycho-Oncology, 18, 1261-1272. https://doi.org/10.1002/pon.1529

Lengacher, C. A., Kip, K. E., Barta, M., Post-White, J., Jacobsen, P. B., Groer, M., et al. (2012). A pilot study evaluating the effect of mindfulness-based stress reduction on psychological status, physical status, salivary cortisol, and interleukin-6 among advanced-stage cancer patients and their caregivers. Journal of Holistic Nursing, 30(3), 170-185.

Lengacher, C. A., Shelton, M. M., Reich, R. R., Barta, M. K., Johnson-Mallard, V., Moscoso, M., et al. (2014). Mindfulness based stress reduction (MBSR(BC)) in breast cancer: evaluating fear of recurrence (FOR) as a mediator of psychological and physical symptoms in a randomized control trial (RCT). Journal of Behavioral Medicine, 37, 185-195. http://dx.doi.org/10.1007/s10865-012-9473-6

Mañas, I. (2009). Mindfulness (atención plena): la meditación en psicología clínica. Gaceta de Psicología, 50, 13-29.

Ministerio de Salud. (2013). Análisis de la situación de cáncer en el Perú. Recuperado de http://www.dge.gob.pe/portal/docs/asis_cancer.pdf

Molina, T. (2009). Psiconeuroinmunoendocrinología. MedULA, 18, 155-164.

Moscoso, M. S. (2009). De la mente a la célula: impacto del estrés en psiconeuroinmunología. Liberabit, 15(2), 143-152. 
Moscoso, M. S. (2010). El estrés crónico y la terapia cognitiva centrada en mindfulness: una nueva dimensión en psiconeuroinmunología. Persona, 13, 11-29.

Moscoso, M. S. (2014). El estudio científico del estrés crónico en neurociencias y psicooncología. Persona, 17, 53-70.

Moscoso, M. S., y Lengacher, C. A. (2015). Mecanismos neurocognitivos de la terapia basada en mindfulness. Liberabit, 21(2), 221-233.

Moscoso, M. S., Lengacher, C. A., y Reheiser, E. C. (2012). The assessment of the perceived emotional distress: the neglected side of cancer care. Journal of Psicooncologia, 9, 277-288.

Moscoso, M. S., Reheiser, E. C., y Hann, D. (2004). Effects of a brief mindfulness-based stress reduction intervention on cancer patients. Psycho-Oncology, 13(1), S12.

Rivera, P., y Vásquez, N. (2013). Percepción de imagen corporal y sentido de vida en mujeres con tratamiento quirúrgico por cáncer de mama (tesis de pregrado). Universidad Católica de Santa María.

Roldán, K., y Torres, C. (2009). Programa de autoconocimiento para reducir los niveles de estrés percibido (tesis de pregrado). Universidad Católica de Santa María.

Sánchez, T. (2015). Efectos psíquicos de la mastectomía (preventiva y terapéutica). Cambios en la percepción de las mujeres con cáncer de mama. Papeles del Psicólogo, 36(1). Recuperado de http://www.papelesdelpsicologo.es

Speca, M., Carlson, L. E., Goodey, E., y Angen, M. (2000). A randomized, wait-list controlled trial: the effect of a mindfulness meditation-based stress reduction program on mood and symptoms of stress in cancer outpatients. Psychosomatic Medicine, 62, 613-622.

Stahl, B., y Goldstein, E. (2010). Mindfulness para reducir el estrés. Una guía práctica. Barcelona: Kairós.

Tacón, A. M., Caldera, Y. M., y Ronaghan, C. (2004). Mindfulness-based stress reduction in women with breast cancer. Families, Systems, \& Health, 22(2), 193-203. http:// dx.doi.org/10.1037/1091-7527.22.2.193

Tateishi, V. (2011). Estilos de humor y sintomatología depresiva en pacientes con cáncer de mama (tesis de pregrado). Pontificia Universidad Católica del Perú.

Teasdale, J. D., Segal, Z. V., Williams, J. M. G., Ridgeway, V., Soulsby, J., y Lau, M. (2000). Prevention of relapse/recurrence in major depression by mindfulness-based cognitive therapy. Journal of Consulting and Clinical Psychology, 68, 615-623. 